\title{
Communication styles of Swedish veterinarians involved in dairy herd health management: A motivational interviewing perspective
}

\author{
C. Svensson, ${ }^{1 *}$ U. Emanuelson, ${ }^{1}$ A. M. Bard, ${ }^{2}$ L. Forsberg, ${ }^{3}$ H. Wickström, ${ }^{4}$ and K. K. Reyher ${ }^{2}$ \\ ${ }^{1}$ Department of Clinical Sciences, Swedish University of Agricultural Sciences, PO Box 7054, SE-750 07 Uppsala, Sweden \\ ${ }^{2}$ Bristol Veterinary School, University of Bristol, Langford House, Langford, North Somerset BS40 5DU, United Kingdom \\ ${ }^{3}$ MICLab AB, Drottninggatan 55, SE-111 21 Stockholm, Sweden \\ ${ }^{4}$ MeetMe Psykologkonsult AB, Åvägen 16, SE-443 31 Lerum, Sweden
}

\section{ABSTRACT}

Veterinary communication has evolved in the context of traditional veterinary tasks, such as diagnosing and treating sick animals, and this may encourage a directive communication style, in which the veterinarian acts as an expert, directing the client in what to do and how to do it. This type of communication style has been shown to evoke resistance to change in clients who are experiencing psychological ambivalence, a well-known aspect of farmers' herd health decisions. Veterinary herd health management (VHHM) constitutes an increasing proportion of the work of cattle veterinarians and often focuses on behavior change. The present study aimed to characterize communication styles of Swedish dairy cattle veterinarians involved in VHHM in relation to their skills in facilitating behavior change. A secondary aim was to investigate whether these skills differed among veterinarians depending on their experience in the veterinary profession or in VHHM. Audio-recorded role-play conversations ( $\mathrm{n}=$ 123; reflecting VHHM telephone consultations) with 42 veterinarians involved in VHHM in Swedish dairy herds and audio-recorded on-farm consultations ( $\mathrm{n}=$ 86) with 18 of those veterinarians were coded using a system developed to evaluate motivational interviewing (MI) skills. Motivational interviewing is a communication methodology aimed at facilitating clients' internal motivation to change. The MI Treatment Integrity (MITI) code identifies frequency counts of 10 verbal behaviors, and assesses 4 global variables on a Likert scale, based on $20 \mathrm{~min}$ of conversation. It also suggests 6 summary measurements of MI competency based on these 14 original variables. Of the 42 veterinarians, 39 also responded to a web questionnaire about their age, continuing education, and experience in the profession, in dairy herds and in VHHM. We analyzed associa-

Received September 21, 2018

Accepted July 19, 2019.

*Corresponding author: catarina.svensson@slu.se tions between the 6 summary MITI variables from the role-play conversations and characteristics of the 39 veterinarians using logistic and linear multivariable regression models. Veterinarians in the role-play and on-farm conversations relied predominantly on giving information, questions, and persuasion in their consultation approaches. They generally did not explore the client's expectations or wishes regarding the agenda for the consultation, or acknowledge the client's right to make decisions about actions. Veterinarians gave advice without exploring the client's need for the advice or how the information was perceived. We found a significant reduction in so-called relational scores (Empathy plus Partnership) and an increase in MI-nonadherent behaviors (Persuasion plus Confront) as years of veterinary experience increased. Results showed that there is room for improvement in the communication style of veterinarians involved in VHHM. Wider literature suggests that training veterinarians in a client-centered communication methodology such as MI may increase the demand for and success of VHHM.

Key words: veterinary herd health management, veterinarian-client communication

\section{INTRODUCTION}

Cattle veterinarians have traditionally focused on the diagnosis and treatment of clinically diseased animals, and these tasks still constitute the major role of many veterinarians (Hall and Wapenaar, 2012). Veterinary communication has evolved in this context, and veterinarian-client conversations generally start by opening the consultation and gathering information, followed by a discussion of examination results and the planning and execution of treatment.

Cattle veterinarians in Sweden and other countries are moving increasingly toward the role of herd health advisor. Although cattle veterinarians have been encouraged for decades to increase their engagement in veterinary herd health management (VHHM; Radostits, 2001; LeBlanc et al., 2006; Duval et al., 
2016), the process has been slow. One obstacle may be a mismatch between current advisory communication and the specific aims of VHHM services, where the focus is largely on behavior change: altering established management routines to prevent clinical and subclinical disease, improve animal welfare, and ensure the profitability of the enterprise. In traditional veterinarian-client conversations, veterinarians typically set the agenda, contribute most to the conversation, and leave the client with a passive role (Shaw et al., 2006). However, giving advice in this persuasive form has been shown to evoke resistance to change in clients who are experiencing psychological ambivalence (Apodaca and Longabaugh, 2009), a recognized trait in farmers' appraisal of complex herd health decisions (Jansen and Lam, 2012). This type of communication style is therefore not likely to improve farmer engagement with VHHM programs. Indeed, farmers report poor communication to be an obstacle to hiring veterinarians for VHHM (Svensson et al., 2018).

In the medical and psychological sciences, practitioners are increasingly adopting an evidence-based methodology called motivational interviewing (MI), a client-centered communication approach that aims to facilitate clients' internal motivation to change (Rollnick and Miller, 1995; Miller and Rollnick, 2012). Strong research evidence supports the efficacy of MI, notably in its application to drug and alcohol abuse counseling but also to other types of behavior change (Hettema et al., 2005; Lundahl et al., 2010). Clients generally trust their own arguments for change more than the arguments of others. In MI methodology, clients' own statements expressing consideration of, motivation for, or commitment to behavior change is called Change Talk, and clients' statements expressing why they may not change is called Sustain Talk. Technical communication skills that enhance Change Talk and soften Sustain Talk are critical in MI, together with relational skills that express empathy, acceptance, and partnership (Miller and Rollnick, 2012). Motivational interviewing has not been used to any significant extent in veterinary medicine in Sweden to this point, and MI training had not been provided to Swedish veterinarians before this study was performed. The extent to which an advisor uses MI can be measured with the Motivational Interviewing Treatment Integrity coding system (MITI; Moyers et al., 2005, 2014), a well-established method of measuring advisor communication skills correlated with client behavior change outcomes (Moyers et al., 2005; 2016). The MITI includes only verbal behaviors; it does not include nonverbal behaviors.

Veterinary communication in the small animal clinical setting has been relatively well described (Shaw et al., 2008; Nogueira Borden et al., 2010; McArthur and Fitzgerald, 2013), but communication research in the large animal setting is still in its infancy. As well, existing literature in small and large animal settings (Jansen, 2010; Derks et al., 2013; Ritter et al., 2018) focuses on classification of communication patterns other than MITI; papers using MITI to assess efficacy in the pursuit of behavior change are rare (Bard, 2018). Given the explicit link of MITI to client change and outcome behaviors (Moyers et al., 2016), its application to onfarm communication merits attention. A better understanding is needed of the status of change-oriented communication skills among veterinarians practicing VHHM to be able to better design and target educational actions aimed at improving efficiency in veterinary advisory services.

It may seem logical that veterinarians with more experience in the profession and VHHM have developed their communication skills to suit an advisory role and facilitate client behavior change. Indeed, communication patterns have been found to differ between veterinarians of different age groups (Ritter et al., 2018). However, research from other professions suggests that experience is insufficient to significantly improve communication skills (Miller and Moyers, 2017). Communication patterns may also differ because of variations in communication training (Shaw et al., 2006; Ritter et al., 2018).

The present study aimed at characterizing the communication styles of Swedish dairy cattle veterinarians involved in VHHM in relation to their skills to stimulate behavior change. A secondary aim was to investigate whether these skills differed between veterinarians depending on their experience in the veterinary profession or in VHHM.

\section{MATERIALS AND METHODS}

Role-play scenarios, reflecting VHHM telephone consultations between cattle veterinarians $(\mathrm{n}=42)$ and professional actors $(\mathrm{n}=3)$, were recorded between March and May 2016. The actors were experienced in role-play scenarios in various settings where MI has been used. The veterinarians were randomized to 2 groups, and 1 group $(\mathrm{n}=21)$ was also requested to record VHHM consultations on farm between June and December 2016. Conversations were recorded using a digital voice recorder or a microphone. Audio files were uploaded to the coding laboratory website (https:// miclab.se), where they were retrieved by MITI coders.

Ethics approval for this study was granted by the Regional Ethical Review Board in Uppsala (reference number 2016/041). 


\section{Participating Veterinarians}

This study was part of a larger project on MI in VHHM, which involved a 6-mo training course in MI and continued efforts over a 2-yr period for participating veterinarians. To allow for sufficient engagement and minimize dropouts, participants were selected from among volunteers whose participation was supported by their employers. To maximize sample size, we invited participants from all main categories of dairy cattle veterinarians involved in VHHM in Sweden. These included practitioners employed by the District Veterinary Organization (Swedish Board of Agriculture); self-employed practitioners; and field veterinarians employed by regional dairy associations. Veterinarians employed by the District Veterinary Organization (and most self-employed veterinarians) provided emergency clinical services as well as VHHM. Most of these veterinarians were also engaged in veterinary services for horses and small animals and offered $24 \mathrm{~h}$ services. The veterinarians at the regional dairy associations $(\mathrm{n}=23$; $87 \%$ female) served larger geographic areas, all worked with cattle only, and generally offered VHHM together with routine services such as udder health examinations, reproductive examinations, and treatments.

District veterinarians involved in VHHM in dairy herds were identified from among those who were taking or had taken a course licensing them to sign contracts with farms related to delegated medicine use in their VHHM work, according to Swedish legislation $(\mathrm{n}=56$; $73 \%$ female). All veterinarians from the regional dairy associations had also undertaken this type of course. Self-employed practitioners involved in VHHM in dairy herds were identified from participants enrolled in the main VHHM network ( $\mathrm{n}=18 ; 83 \%$ female).

Information about the project was provided to veterinarians' employers (the District Veterinary Organization and regional dairy associations), who were asked about their interest in allowing veterinarians to join the project. Lists of veterinarians allowed to join were provided by the employers; the listed veterinarians were given information about the project via telephone and invited to participate. Self-employed cattle practitioners enrolled in the VHHM network received information about the project and an invitation via e-mail. To support the engagement of veterinarians in the larger project, we included an introductory information session (5-10 min) about MI in the presentation about the project. In total, 42 veterinarians (25 from the District Veterinary Organization, 11 from regional dairy associations, and 6 self-employed; i.e., approximately $43 \%$ of all Swedish veterinarians working in dairy herds with VHHM) volunteered for the study. Of those, $93 \%$ were female.
Before the role-play sessions, participants consented to the use of session recordings and completed a webbased questionnaire developed using Netigate (https: //www.netigate.net/sv/). The questionnaire detailed the participant's year of birth and year of graduation as a veterinarian, the number of years they had been a veterinarian, the number of years they had been a dairy veterinarian, and any continuing education in cattle medicine (as measures of their experience as a veterinarian). Participants also indicated the number of years they had been participating in VHHM, the proportion of VHHM in their services, and any previous continuing education in VHHM (as measures of their experience in VHHM) as well as previous continuing education in communication. Because the information was considered difficult to estimate precisely, the questionnaire used prespecified categories (see Data Editing and Statistical Analysis) for all questions except year of birth and graduation.

\section{Role Play}

Actors were not given a script; instead, they received a farm profile (herd size, productivity, disease situation, current management routines) and background information to shape a character (a famer, farm manager, or animal caretaker) reflecting typical Swedish situations. Each actor played the character for only one scenario. To form their character, actors received information about the character's age and everyday life, their job tasks and role on the farm, their family situation, perceived barriers to uptake of advice and attitudes/ norms/perceived control of the particular herd health problem on their farm. The actors were instructed to improvise during each interaction, responding to communication by the veterinarians in an appropriate and genuine manner given their assumed character, as a means of generating an authentic simulation of the veterinarian-client encounter.

Because the actors were previously unfamiliar with dairy farming, they underwent training before the sessions that highlighted traditional vocabulary, practical farm routines, disease problems, and preventive actions relating to their character. The actors studied dairy farm press articles and material illustrating life on dairy farms and typical vocabulary for veterinarians and farmers/farm staff. They also interviewed a dairy farmer or employee for their character and studied specific information about the herd health problems their character was consulting the veterinarian for. The role plays were piloted with the first author (a cattle veterinarian with experience in VHHM) and each actor in advance of data collection, and the knowledge and vocabulary of the actors were discussed. 
The scenarios were constructed in collaboration between the authors, a farmer, and the 3 actors using experience from similar published role-play sessions (Bard et al., 2017), and they were translated from real advisory situations. All 3 actors were women. The scenarios were (1) increased occurrence of displaced abomasum in a herd with a history of several cases of hypocalcemia, discussed with a female dairy farmer; (2) udder health problems, discussed with a farm manager working at a farm that used to belong to her parents and now belongs to her brother; and (3) a calf diarrhea problem, discussed with a female calf caretaker.

Because all role plays were conducted via telephone for practical reasons, scenarios depicted telephone consultations with a client who the veterinarian had met on the farm when time was restricted and who had agreed to continue the discussion over the telephone. This was representative of real-life situations; Swedish dairy cattle veterinarians provide telephone consultations on a regular basis.

Participating veterinarians received general information about how the role-play sessions were set up, that the sessions were to be initiated by the actors, and that the actor would improvise a natural closing of the interaction after $20 \mathrm{~min}$ of conversation. Furthermore, veterinarians were told that their communication skills were the focus of the role plays, not their technical skills, and that they would be provided with a list of 3 to 4 relevant preventive measures to use when they formed their advice. Coding sequences of conversation longer than 20 min can reduce reliability, so the length of the conversations was chosen to optimize the conditions for reliable MITI coding, according to Moyers et al. (2014).

The restriction to use only the provided preventive measures in the veterinarians' recommendations was intended to standardize conditions for the communication - thereby reducing variability related to context - and to make the situation less stressful for the veterinarians, enabling them to concentrate on their communication. Veterinarians received detailed information via e-mail about the farm (including the health problem the consultation was to address and management routines important for this problem) 10 min before they were called by the actor. Each veterinarian performed 3 conversations: 1 with each of the 3 actors and their topics. The veterinarians booked their 3 sessions in a digital booking system according to their availability and that of the actors over 3 mo of scheduled appointments.

\section{On-Farm VHHM Recordings}

Each veterinarian was asked to choose a convenience sample of farms from among their clients and record
VHHM conversations on 5 dairy farms. Inclusion criteria were that it would be possible to specify the preventive actions discussed during the visit in a document (health plan), and that the farm had an interest in following up this health plan during a later visit. These criteria were related to aims for other parts of the project.

Veterinarians discussed the purpose and design of the project with farm owners and staff and invited them to participate. Farmers and staff who agreed to participate provided written consent to share the recordings. Veterinarians were asked to record a minimum of 20 min of conversation. In cases of longer conversations, they could record only the 20 min they considered most relevant to discussions of improving animal health or they could record the whole conversation and inform the coding laboratory of which parts they considered most relevant.

\section{Coding of Communication Styles}

Coding of audio-recorded conversations was conducted by 3 professional coders at the MI coding laboratory MICLab AB (Stockholm) according to the MITI 4.2.1 manual (Moyers et al., 2014) translated into Swedish). All role-play recordings had been terminated by the actors after approximately $20 \mathrm{~min}$, and the full $20 \mathrm{~min}$ of each conversation was coded. For on-farm recordings, 20 min of each conversation was coded, according to the information provided by the veterinarian. If the veterinarian indicated that more than 20 min of conversation dealt with actions to improve herd health, the coder chose a random segment of $20 \mathrm{~min}$. Recordings were encrypted during uploading to the MICLab homepage and registered in a database on a protected server. Coders did not know the identities of the veterinarians or actors. To sustain competence, coders at MICLab participate in a quality assurance program. The program comprises weekly training sessions based on independently coded recordings. Furthermore, inter-rater reliability tests are performed twice a year. The test related to the coding in the present study was performed in June 2017, and found that the intra-class correlations of the different MITI variables was between 0.61 and 0.97. A word list was developed to facilitate coders' understanding of the dairy farm-veterinarian context. Before coding the conversations, the 3 coders received training by the first author and studied transcripts of farmer conversations and written material illustrating typical veterinary and farmer/farm staff vocabulary. The MITI 4.2.1 coding manual (Moyers et al., 2014) identifies frequency counts of 10 verbal behaviors, and assessments of 4 global scores on a Likert scale ranging from 1 (low) to 5 (high; Table 1) based on 20 min of 
Table 1. Brief description of the MITI 4 variables used in the assessment of motivational interviewing skills ${ }^{1,2}$

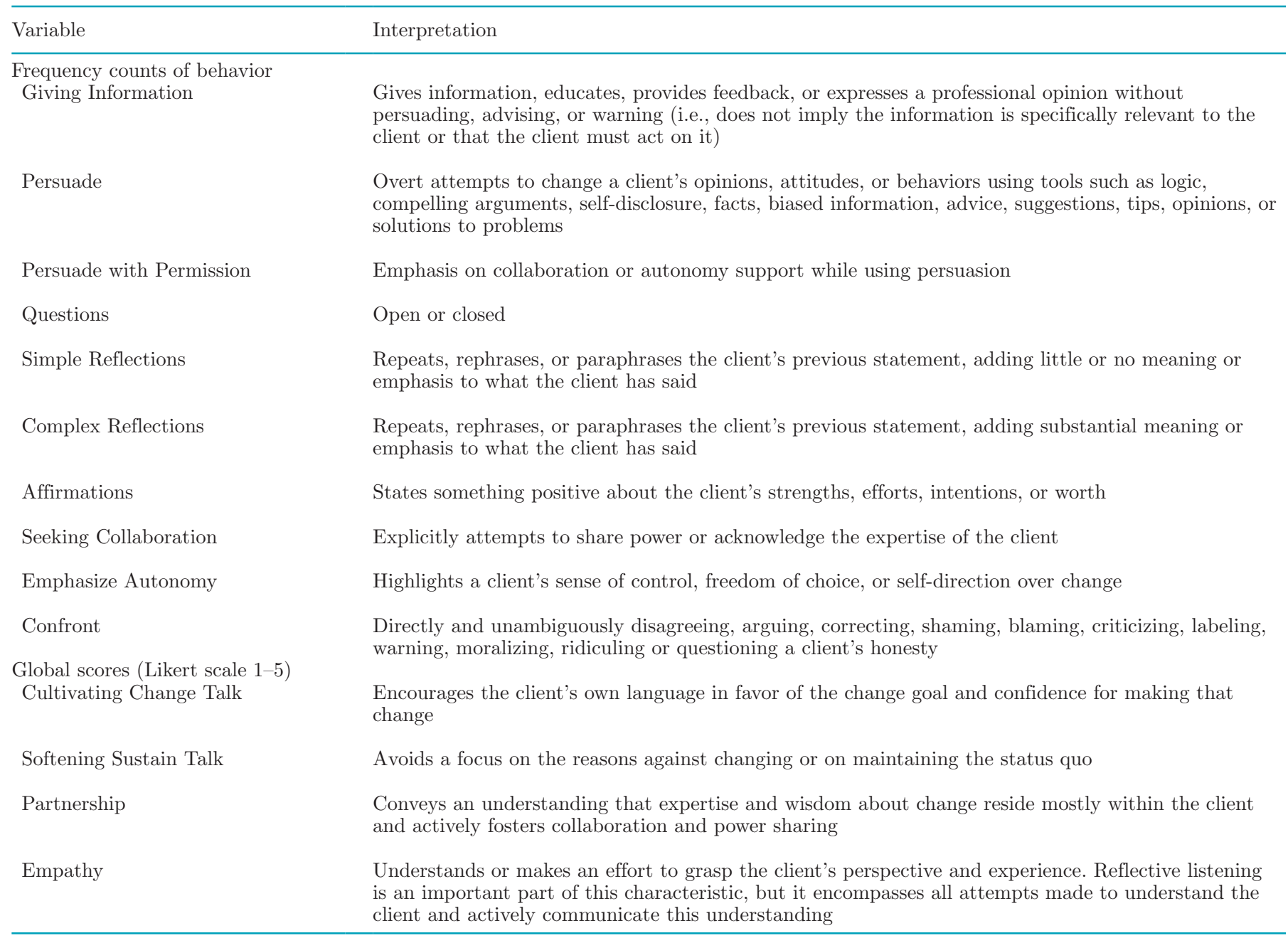

${ }^{1}$ MITI = Motivational Interviewing Treatment Integrity.

${ }^{2}$ Moyers et al., 2014.

conversation. Besides word content, tone of voice, volume, and tempo are also evaluated. Verbal behaviors included in the frequency count are Giving Information, Persuade, Persuade with Permission, Questions, Simple Reflections, Complex Reflections, Affirmations, Seeking Collaboration, Emphasize Autonomy, and Confront. Global scores are the extent to which the veterinarian (1) actively encourages the client's own language in favor of the VHHM change goal and their confidence in making the change (Cultivating Change Talk); (2) avoids focusing on the client's reasons for not changing their herd health management or maintaining the status quo on farm (Softening Sustain Talk); (2) actively fosters collaboration and power-sharing with the client (Partnership); and (4) understands or makes an active effort to grasp the client's perspective and experience (Empathy) during the VHHM interaction (Table 1).
The MITI 4.2.1 coding manual also specifies 6 summary measurements (Relational, Technical, MI-Nonadherent Behaviors, MI-Adherent Behaviors, Reflectionsto-Question Ratio, Percent Complex Reflections) derived from the 14 original variables (Moyers et al., 2014) and these were calculated for each recording. The 6 summary measurements were calculated according to the following formulas:

$$
\text { Relational }=(\text { Partnership }+ \text { Empathy }) / 2 ;
$$

Technical $=($ Cultivating Change Talk + Softening $) / 2$;

MI-Nonadherent Behaviors = Persuade + Confront;

MI-Adherent Behaviors = Emphasize Autonomy

$$
+ \text { Seek Collaboration + Affirm; }
$$


Reflections-to-Question Ratio $=($ Simple Reflections

+ Complex Reflections)/Total Questions; and

Percent Complex Reflections $=$ Complex Reflections $/$

(Simple Reflections + Complex Reflections).

As suggested by Moyers et al. (2014), the summary measurements were further categorized into fair and good competency, respectively, using the following thresholds: Relational $\geq 3.5$ and $\geq 4$; Technical $\geq 3$ and $\geq 4$; Reflections-to-Question Ratio $\geq 1: 1$ and $\geq 2: 1$; and Percent Complex Reflections $\geq 40 \%$ and $\geq 50 \%$.

\section{Data Editing and Statistical Analysis}

Because of personal constraints, 1 veterinarian carried out role-play sessions with only 2 of the 3 actors. Audio-recordings from 2 sessions involving 2 veterinarians and 2 different actors were not successfully uploaded to the laboratory website. Hence, the total role-play material consisted of 123 audio-recorded role-play conversations with 42 veterinarians; these data were used in the descriptive statistics. Two veterinarians did not answer the web questionnaire, and 1 veterinarian had a missing value with respect to percentage VHHM in the web questionnaire. Data from the recordings of these 3 veterinarians ( 8 in total: 3 from 2 veterinarians and 2 from the veterinarian who completed only 2 sessions) were included in the descriptive statistics but not in the multivariable statistical analyses. The multivariable analysis thus included 115 role-play recordings from 39 veterinarians. Because of time constraints, change of position, and maternity leave, respectively, 3 of the 21 veterinarians in the selected group failed to record any on-farm conversations; 1 veterinarian recorded only 2 conversations, and 3 veterinarians recorded only 4 conversations. Three of the veterinarians recorded 6 conversations each. Hence, the total on-farm material consisted of 86 recordings from 18 veterinarians. Seven recordings were shorter than $20 \mathrm{~min}$ and, for these, frequency counts were adjusted to reflect a $20 \mathrm{~min}$ recording.

For each of the 42 and 18 veterinarians, respectively, we calculated a mean value from the 2 to 3 available recordings for each MITI variable and each of the 6 summary MITI measurements. These were then used to calculate descriptive statistics using Excel (2016; Microsoft Corp., Redmond, WA).

Multivariable regression models, using all available role-play recordings, were run to assess associations between the characteristics of the veterinarians and their communication skills, as expressed by the 6 summary
MITI measurements. A random effect of veterinarian was modeled but could not be included because of convergence problems. Linear regression models were used for Relational, Technical, MI-Nonadherent Behaviors and MI-Adherent Behaviors, and logistic regression models were used to analyze fair vs. not fair competency for Reflections-to-Question Ratio and Percent Complex Reflections. Models included the following as fixed effects: years as veterinarian (continuous), years as a veterinarian in dairy herds $(1-5,5-15,>15)$, years in VHHM $(<1,1-5,5-15,>15)$, volume of VHHM $(0-10 \%, 11-25 \%, 26-50 \%>50 \%$ of working time), continuing education in cattle medicine or VHHM (single day, 1-2 d, >2 d), education in communication (none, a few hours, $1 \mathrm{~d},>1 \mathrm{~d}$ ) and actor. We assessed multicollinearity between the explanatory variables by variance inflation factors, but no multicollinearity was present. We checked the goodness-of-fit of the linear regression models by graphically assessing the residuals for normal distribution using quantile-quantile plots and of the logistic regression models using the Hosmer-Lemeshow test; all models showed satisfactory fit. All statistical analyses were performed using SAS version 9.4 (SAS Institute Inc., Cary, NC).

\section{RESULTS}

In both role-play and on-farm recordings, veterinarians relied predominantly on Questions, Giving Information, and Persuasion in their consultation approaches (Table 2 ), meaning that the advisory interactions focused on eliciting information from the client, providing information/education to the client and making overt attempts to change the client's opinions, behavior, or attitudes related to implementing preventive measures against disease complexes. The veterinarians generally made little effort to elicit the client's expectations or wishes for the agenda of the consultation (Seeking Collaboration; Table 2). This meant that the veterinarians set the agenda and controlled the conversations, and when they recommended preventive actions to the clients and explained why these should be implemented, veterinarians made few attempts to explore the client's need for advice or how the advice was perceived. Similarly, they made few attempts to acknowledge the client's freedom of choice and right to make decisions about their animals and their farm (Emphasize Autonomy; Table 2 ); instead, they tried to persuade them to take the actions the veterinarians perceived to be most suitable based on their expertise. Veterinarians largely avoided confrontation (Confront); the majority [29 (71\%) role play; $10(55 \%)$ on farm] had no Confront behaviors at all. They made few positive statements confirming the 
client's behavior, attitude, and intentions (Affirmation; Table 2). They seldom demonstrated active listening by forming conjectures about what the client thought and felt (Reflections; Table 2); however, $5(12 \%)$ of the veterinarians reached fair competency with respect to Reflection-to-Question Ratio ( $\geq 1: 1)$ and $1(2 \%)$ reached good competency $(\geq 2: 1)$ in the role-play conversations. In the on-farm conversations, the veterinarians used slightly more Simple Reflections, but the proportions reaching fair $(17 \%)$ and good $(0 \%)$ competency for Reflections-to-Question Ratio were similar.

With respect to global scores, a minority of the veterinarians $[12(29 \%)]$ actively expressed their interest in and acknowledged the importance of the client's views and expertise (Partnership >3.0) in the role-play conversations, but none of them did so in the on-farm conversations. Similarly, a minority [6 (15\%); on farm $1,5 \%$ of the veterinarians actively communicated an understanding and effort to grasp the client's perspective and experience (Empathy >3.0). Few veterinarians showed effort in exploring the client's own reasons for taking action (Cultivating Change Talk; Table 2). Veterinarians generally refrained from giving focus to reasons against taking action or in favor of the status quo (Softening Sustain Talk; Table 2). Five veterinarians $(12 \%)$ reached fair competency with respect to their skills in the summary measurement Relational, and 27 veterinarians $(64 \%)$ reached fair $(\geq 3.5)$ and 1 good $(\geq 4)$ competency with respect to Technical scores in the role-play conversations; none of the veterinarians reached those levels in the on-farm conversations.

Descriptive statistics according to the characteristics of the veterinarians participating in the role-play sessions are shown in Supplemental Table S1 (http://doi .org/10.3168/jds.2018-15731). Three (7.5\%) of the 40 veterinarians who completed the questionnaire were men, and the rest were women; their ages ranged from 28 to 60 years (mean 42, standard deviation 9.6). Veterinarians had been in the profession for 2 to 32 years (median 9; 25th-75th percentile 6-25). Seventy percent had worked in dairy herds for 5 years or more, but $50 \%$ had worked for $1-5$ years with VHHM. All veterinarians had attended continuing education courses on cattle medicine, but few had taken longer continuing courses in communication (Supplemental Table S1). Results from the multivariable regression analyses of associations between the characteristics of veterinarians and their skills in the summary measurements Relational

Table 2. Descriptive statistics for 14 original variables (frequency counts and global scores) and 6 summary variables describing motivational interviewing skills

\begin{tabular}{|c|c|c|}
\hline \multirow[b]{2}{*}{ Variable } & \multicolumn{2}{|c|}{ Mean (SD); 25 th to 75 th percentile } \\
\hline & Role play $^{1}$ & On farm ${ }^{2}$ \\
\hline \multicolumn{3}{|c|}{ Frequency counts (per 20-min conversation) } \\
\hline Giving Information & $15.2(3.43) ; 12.4-17.6$ & $20.6(5.00) ; 18.1-23.9$ \\
\hline Persuade & $9.3(3.39): 7.4-10.9$ & $6.2(2.92): 4.0-7.6$ \\
\hline Persuade with Permission & $1.8(1.09) ; 1.0-2.6$ & $0.9(0.66) ; 0.3-1.4$ \\
\hline Questions & $8.3(4.02) ; 5.4-10.9$ & $13.4(4.92) ; 10.5-15.8$ \\
\hline Simple Reflections & $2.1(1.50) ; 1.0-2.7$ & $5.1(3.20) ; 2.4-6.8$ \\
\hline Complex Reflections & $2.0(1.26) ; 1.0-2.6$ & $2.4(1.66) ; 1.4-3.0$ \\
\hline Affirmations & $1.4(0.99) ; 0.7-2.0$ & $2.3(1.66) ; 1.4-3.0$ \\
\hline Seeking Collaboration & $2.6(1.35) ; 1.7-3.3$ & $0.4(0.49) ; 0.0-0.5$ \\
\hline Emphasize Autonomy & $0.2(0.34) ; 0.0-0.3$ & $0.1(0.20) ; 0.0-0.1$ \\
\hline Confront & $0.2(0.35) ; 0.0-0.3$ & $0.2(0.24) ; 0.0-0.2$ \\
\hline \multicolumn{3}{|l|}{ Global scores (Likert scale 1-5) } \\
\hline Cultivating Change Talk & $2.4(0.55) ; 2.0-2.7$ & $1.4(0.46) ; 1.0-1.8$ \\
\hline Softening Sustain Talk & $3.7(0.31) ; 3.7-4.0$ & $3.1(0.43) ; 3.0-3.3$ \\
\hline Partnership & $2.8(0.60) ; 2.3-3.3$ & $1.7(0.60) ; 1.2-2.0$ \\
\hline Empathy & $2.5(0.62) ; 2.0-3.0$ & $1.8(0.56) ; 1.5-2.0$ \\
\hline \multicolumn{3}{|l|}{ Summary variables } \\
\hline Relational $^{3}$ & $2.7(0.56) ; 2.2-3.2$ & $1.8(0.56) ; 1.3-2.0$ \\
\hline Technical $^{4}$ & $3.1(0.57) ; 2.8-3.3$ & $2.2(0.41) ; 2.0-2.5$ \\
\hline MI-adherent behaviors ${ }^{5}$ & $4.3(2.09) ; 2.4-5.7$ & $2.9(2.01) ; 1.6-3.5$ \\
\hline MI-nonadherent behaviors ${ }^{6}$ & $9.5(3.49) ; 7.4-11.2$ & $6.4(2.95) ; 4.0-8.2$ \\
\hline Reflection-to-Question Ratio & $0.7(0.63) ; 0.3-0.8$ & $0.7(0.38) ; 0.5-0.8$ \\
\hline Percent Complex Reflections & $0.4(0.21) ; 0.3-0.6$ & $0.3(0.15) ; 0.2-0.4$ \\
\hline
\end{tabular}


and Technical MI skills are shown in Supplemental Table S2 (http://doi.org/10.3168/jds.2018-15731). Associations with the summary measurements MINonadherent Behaviors and MI-Adherent Behaviors are shown in Table 3. We found no effects of experience, including continuing education, on the summary measurements Reflection-to-Question Ratio, Percent Complex Reflections (data not shown), or Technical skills (Supplemental Table S2). We found statistically significant associations between Relational skills and experience in VHHM (years in VHHM, proportion of VHHM, and communication training). The lowest skills were identified in veterinarians with the most years in VHHM, but also in those who had the lowest proportion of their work in VHHM. MI-Nonadherent Behaviors increased with number of years as a veterinarian. We found variable associations between communication training and MI-Adherent Behaviors, but veterinarians with only a few hours of training had significantly fewer MI-Nonadherent Behaviors. Variable associations were also found between the proportion of work that was VHHM and MI-Adherent Behaviors. We found a significant effect of the actor on Relational skills, MINonadherent Behaviors and MI-Adherent Behaviors (Table 3 and Supplemental Table S2).

\section{DISCUSSION}

\section{Characterization of Communication Styles}

In their consultation approaches in both role-play sessions and on-farm VHHM consultations, veterinarians relied predominantly on Giving Information, Questions and Persuasion. Veterinarians controlled the conversations and showed little solicitation of client opinion. This was in accordance with the findings of others (Shaw et al., 2006; Jansen, 2010; Bard, 2018, Ritter et al., 2018) and suggests that these veterinarians adopted a paternalistic communication style, taking on the role of an expert who determines the client's best decisions based on an assumption of shared objectives. Such a communication style may give the client the impression that the advisor is not treating them as an equal partner in discussions, does not take their expertise into consideration, and does not fully accept their right to make the decisions in matters that are important to them (Miller and Rollnick, 2012). This may counteract the purpose of the conversation, rendering the client less interested in the veterinarian's information and recommendations and negatively affecting the working relationship between veterinarian and client. In contrast, previous work with Swedish farmers and farm managers showed that they stressed the importance of being acknowledged for their competence and recog- nized for making decisions on their farms (Svensson et al., 2018).

The need for a shared understanding (between veterinarian and farmer) of the farmer's priorities has also been identified as a critical way to underpin the working relationship and support the enactment of herd health advice (Bard, 2018). This shared understanding may be thwarted by a communication style such as the one adopted by the veterinarians in the present study, which left the client in a passive role as veterinarians evoked and explored the true perspectives of their clients poorly. Indeed, it has also been reported that veterinarians seldom investigate farmer goals and motivations and therefore come to incorrect conclusions about farmer priorities (Kristensen and Enevoldsen, 2008; Jansen et al., 2010; Derks et al., 2013). Ritter et al. (2018) calculated the relationship-centeredness care score of Canadian dairy veterinarians in VHHM consultancies and reported these to be relatively low. Farmers and farm managers report that a good veterinarian-client relationship is crucial in VHHM (Svensson et al., 2018; Bard, 2018), but it seems reasonable to speculate that the communication style adopted in the consultations reported here may negatively affect engagement with veterinary advice. However, there is currently no consensus about the perfect communication style in VHHM, and additional research is needed to determine which strategy is best suited to encouraging preventive actions under various conditions.

The MITI manuals are updated continuously, and MITI 4 (Moyers et al., 2014) has not yet been used extensively in research. Comparisons with older versions of MITI that have a more extensive evidence base, such as MITI 3.1 (Moyers et al., 2010), are not straightforward. However, the frequencies and global scores we found seem to be comparable in many respects to those reported for other groups of professionals that have not received specific MI training. The predominance of MI-Nonadherent Behaviors in the present study was similar to that reported in studies of health, food safety, and environmental inspectors (Forsberg et al., 2014b; Wickström et al., 2017); social workers and health care personnel (Forsberg et al., 2014a); and British veterinarians (Bard, 2018). Although they used different definitions from those in MI, Ritter et al. (2018) reported that empathy statements were rare in VHHM conversation on dairy farms. Global scores for Empathy were within the same range as for social workers and health care personnel studied by Forsberg et al. (2014a). A directive communication style is by no means unique to veterinary professionals; across diverse professional roles, consulting is often based on the idea that clients lack information and it is the advisors' task to provide solutions and arguments (Miller and Rollnick, 
VETERINARY COMMUNICATION IN HERD HEALTH MANAGEMENT

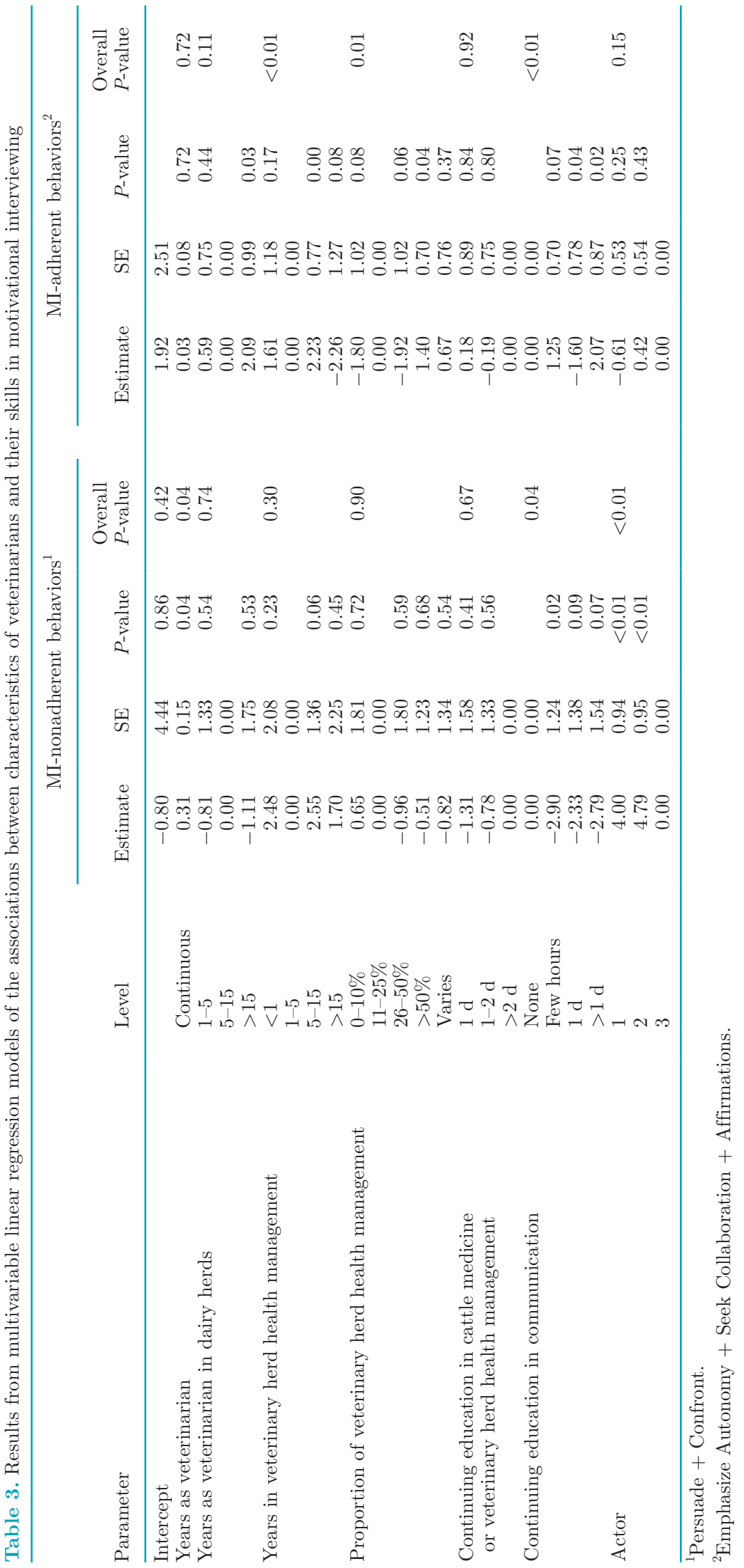


2012), with negative consequences for implementation of the solutions presented. Indeed, the extensive proliferation and success of MI may be in response to these negative consequences: "MI directly addresses what is a very common and often frustrating issue in practice: people's reluctance to change despite advice to do so" (Miller and Moyers, 2017). By focusing on how advice is communicated by advisors and shifting the style of conversation to one that is evocative, collaborative, and client-centered, MI aims to facilitate improvements in both advisor-client relationships and client behavior (i.e., adherence to advice). Further studies are needed to explore client satisfaction and adherence from veterinary consultations using MI methodology.

Skills characteristic of MI, such as evoking client reasons for change (Cultivating Change Talk) and the active listening skill of using Reflections, are not used regularly in everyday or professional conversations (Miller and Rollnick, 2012). It is understandable, therefore, that the estimates for these parameters were low. Simple Reflections were used to a greater extent on farm than in the role-play sessions. Further studies are needed to explore the differences between these 2 settings.

The Partnership scores in the present study were also higher than those of British veterinarians studied by Bard (2018), a difference that may be related to the selection of participants. The participants in the present study were selected based on specific competencies in VHHM work or enrollment in a VHHM network; in Sweden, courses in VHHM often discuss the importance of collaboration and a client-oriented approach (although not, as yet, using MI techniques). No such criteria were used for selecting the British veterinarians. Before the Swedish veterinarians joined the project, they had also received a short (5-10 min) introduction to MI as a concept. In this, MI was described as a guiding, clientcentered communication methodology, establishing a work alliance with the client as one important factor and eliciting clients' own motivation as another. The participants may have concluded, from this information, that partnership was an important aspect and adjusted their communication style as a consequence, into one expressing more partnership than they would otherwise have used. However, previous studies have shown that MI skills require substantial time to learn and that few are capable of learning MI simply by reading or hearing about it; mastery of MI techniques needs long-lasting practical training with feedback (Forsberg et al., 2014a; Wickström et al., 2017; Miller and Moyers, 2017). Therefore, the introductory information did not likely have had a large effect on the results, although smaller effects (e.g., on Partnership) cannot be excluded. Because communication training was offered as part of the project, the participants in the present study might have been more interested in communication than a randomly selected group of dairy cattle veterinarians involved in VHHM. It cannot be ruled out that this interest also meant they may have been more client-oriented in their advisory approach and hence had higher scores for Partnership. Benchmarking of communication skills in cattle veterinarians could be useful and could help improve skills and educational programs, but more research is needed.

Women dominate among Swedish dairy cattle veterinarians, and the proportion of female participants in the present study was slightly higher than in the reference population. Because this has not been well studied, the literature offers little insight into how gender may have affected the results. For instance, a higher proportion of male participants may have resulted in lower scores for Partnership or Empathy.

\section{Associations with Experience Including Continuing Education}

The communication style of veterinarians in this sample was not homogeneous; examination of the associations between veterinarian characteristics and their communication skills highlighted meaningful differences in MITI score attribution. We found a significant effect of experience in VHHM on Relational scores (Empathy plus Partnership). However, longer experience was not associated with higher Relational scores. In contrast, veterinarians recently introduced to VHHM (less than 1 yr) had the highest Relational scores. Similarly, the summary measurement MI-Nonadherent Behaviors (Persuasion plus Confront) increased with years of experience as a veterinarian. These findings might have been because veterinarians primarily perceived their role as one of imparting expertise and instructing the farmer in the management and alleviation of herd health problems (Bard et al., 2017), with delivery of such instructions becoming more directive over time as personal confidence grows. This possibility is supported by the findings of Ritter et al. (2018) that older veterinarians educated and counseled farmers more than younger veterinarians. On the other hand, veterinarians with a very low volume of VHHM (and therefore likely less consistent and ongoing engagement in VHHM) had significantly lower Relational scores than the majority of the participants, who estimated that they had 11 to $25 \%$ of their work hours as VHHM. Further research is needed to clarify the reasons for the differences in communication patterns.

With regards to communication training exposure, we found no effects of experience or continuing education on Technical scores (Supplemental Table S2; http: 
//doi.org/10.3168/jds.2018-15731) and on Reflectionto-Question Ratio or Percent Complex Reflections (data not shown). It is well recognized that MI communication skills are not easy to manifest in communication interactions without specific training (Miller and Rollnick, 2009; Miller and Moyers, 2017). This means that non-MI-specific communication training is unlikely to enhance the complex skills of recognizing and responding to change language (Technical score) and advanced active listening (Reflection-to-Question Ratio, Percent Complex Reflections) measured here. However, we found a significant effect of communication education on MI-Nonadherent Behaviors, with more training reducing their likelihood. It is possible that this skill-refraining from pushing, persuading, and confronting clients - is more readily integrated into non-MI-specific training experiences aiming to enhance the nature of veterinarian-client interactions. Also of interest is the finding that even brief education of only a few hours, which was not specifically MI-oriented, generated less MI-Nonadherent Behavior than no communication education, suggesting that communication training can have measurable effect(s) even over small time frames. Bard et al. (2018) confirmed this finding, demonstrating significant effects on Relational, Technical and active listening behaviors after only a short $(4$ to $5 \mathrm{~h}$ ) introduction to MI with veterinarians. The variable association between communication education and MI-Adherent Behaviors is somewhat more difficult to explain, but might be due to the type of education received. Unfortunately, no details about the type or quality of previous communication education were recorded.

Training in MI would be most likely to improve the skills measured here (because they are considered very important in MI), but other types of communication training may not necessarily discuss or highlight these skills in the same specific way (Miller and Rollnick, 2009). As previously mentioned, no MI training had been provided to veterinarians in Sweden when this study was performed. Ritter et al. (2018) found that veterinarians with 4 years of communication training during veterinary school and veterinarians with no communication training displayed very similar communication patterns in VHHM consultancies.

The employment situation of the veterinarians (i.e., who paid their salary) may affect their skills, but we did not investigate this in the present study because the sample included too few self-employed veterinarians (n $=6$ ) to allow a stable statistical analysis. However, this would be an interesting subject for future research.

We identified a significant effect of actor on the MITI summary measurements of MI-Nonadherent Behaviors and Relational scores, with actor 3 receiving lower Per- suade and Confront scores and higher Empathy and Partnership scores than actors 1 and 2. The differences could have been a synergy of actor presentation and veterinarian response. If actors 2 and 3 played their scenario clients in a differing motivational state than actor 1 (for example, offering relatively more Sustain Talk by comparison), it is reasonable to posit that veterinarians may have similarly adjusted their communication and shifted their communication style (for example, to being more persuasive in the face of Sustain Talk). Additionally, the 3 clients in these role plays had differing roles on the farm (farm manager, farmer, calf caretaker). This may also have evoked differing communication styles from the veterinarians, as they addressed each client depending on the veterinarians' experience and expectation of the differing client roles. Although it is impossible to determine what caused this actor effect, in practice veterinarians meet clients who vary in their disposition, change topics, and farm roles, and the situation in the present study with 3 different actors and scenarios is likely to mimic real situations. Ritter et al. (2018) also reported that communication patterns varied considerably for a single veterinarian interacting with different clients.

\section{Additional Methodological Considerations}

Professional actors are increasingly being used in education and research (Bard, 2018) and to evaluate therapist behavior (Imel et al., 2014). Role play provides "a variety of naturally occurring data [that] is therefore worthy of study" (Seale et al., 2007), which increases confidence in these results despite implicit variability. In MI society, it is often recommended that trainer aspirants use role play to show that they have sufficient MI skills to merit their inclusion in future trainer programs. Role play has several advantages over real on-farm situations. In the present study, the use of the same 3 role-play scenarios for all participants standardized the conditions compared with using real on-farm conversations, which would have produced different conditions for each veterinarian.

The availability of actors may be a limitation when using role play. This was why all 3 actors in the present study were female, whereas in real situations most farmers in Sweden are male. Shaw et al. (2006) reported increased use of biomedical communication patterns in conversations between veterinarians and clients of different sexes, and Ritter et al. (2018) found higher relationship-centered care scores in VHHM conversations with male concordance (i.e., when both veterinarian and client were male). How the high female concordance in the present study may have affected the results is difficult to say, because literature on gender 
aspects of communication skills measured by MITI is scarce.

An advisor's MITI scores may vary not only according to different contexts but also according to his or her "daily fitness." With 3 recordings (and situations) for each veterinarian, the estimate of his or her true MI skill was improved compared with using only 1 recording.

\section{CONCLUSIONS}

In this examination of communication styles in Swedish veterinarians involved in herd health management, veterinarians predominantly relied on Giving Information, Questions and Persuasion with minimal soliciting of client opinion. This style signals an unequal power distribution between veterinarian and client, with the former being the one not only in control of the conversation but also supposing and communicating to have the best solutions to the client's problem. Previous research has identified that such an approach may negatively affect the advisor-client relationship and impair uptake of advice, and that more client-centered communication may better facilitate behavior change. Although we found some evidence that veterinarians became more directive over time, communication style was largely not associated with experience in the veterinary profession or in VHHM. Based on the wider literature, we therefore suggest that targeted education and training in a client-centered communication methodology such as MI at all career stages may be a suitable approach to meeting the needs of the evolving veterinary profession in providing successful VHHM programs aimed at inspiring farmer behavior changes.

\section{ACKNOWLEDGMENTS}

This study was funded by The Swedish Research Council for Environment, Agricultural Sciences and Spatial Planning. The authors thank the participating veterinarians for their time and efforts in performing the role-play sessions. The authors are grateful to Åsa Karlin, Nanny Nilsson, and Emilia Roosmann (MICLab AB, Stockholm, Sweden) for their acting performances and to Mahlena Wiveson and Helena Chaomar (MICLab AB) for coding the recordings.

\section{REFERENCES}

Apodaca, T. R., and R. Longabaugh. 2009. Mechanisms of change in motivational interviewing: A review and preliminary evaluation of the evidence. Addiction 104:705-715. https://doi.org/10.1111/j .1360-0443.2009.02527.x.
Bard, A. 2018. Improving dairy cattle welfare: Examining motivational interviewing, veterinary communication and the herd health advisory paradigm. PhD Thesis. University of Bristol Veterinary School, Bristol, UK.

Bard, A. M., D. C. J. Main, A. M. Haase, H. R. Whay, E. J. Roe, and K. K. Reyher. 2017. The future of veterinary communication: Partnership or persuasion? A qualitative investigation of veterinary communication in the pursuit of client behavior change. PLoS One 12:e0171380. https://doi.org/10.1371/journal.pone.0171380.

Nogueira Borden, L. J., C. L. Adams, B. N. Bonnett, J. R. Shaw, and C. S. Ribble. 2010. Use of the measure of patient-centered communication to analyze euthanasia discussions in companion animal practice. J. Am. Vet. Med. Assoc. 237:1275-1287.

Derks, M., B. van Woudenbergh, M. Boender, W. Kremer, T. van Werven, and H. Hogeveen. 2013. Veterinarian awareness of farmer goals and attitudes to herd health management in The Netherlands. Vet. J. 198:224-228. https://doi.org/10.1016/j.tvj1.2013.07 .018 .

Duval, J. E., N. Bareille, C. Fouricon, A. Madouasse, and M. Vaarst. 2016. Perceptions of French private veterinary practitioners' on their role in organic dairy farms and opportunities to improve their advisory services for organic dairy farmers. Prev. Vet. Med. 133:10-21. https://doi.org/10.1016/j.prevetmed.2016.09.008.

Forsberg, L., M. Beckman, A. Ghaderi, H. Lindqvist, L. Öhman, and K. Börjesson-Toth. 2014a. Utvärdering och utveckling av utbildningar i Motiverande samtal inom hälso-och sjukvården [Evaluation and development of education in motivational inteviewing in health and medical care] Karolinska Institute, Solna, Sweden. [In Swedish]

Forsberg, L., H. Wickström, and H. Källmén. 2014b. Motivational interviewing may facilitate professional interactions with inspectees during environmental inspection and enforcement conversations. PeerJ 2:e508. https://doi.org/10.7717/peerj.508.

Hall, J., and W. Wapenaar. 2012. Opinions and practices of veterinarians and dairy farmers towards herd health management in the UK. Vet. Rec. 170:441-445. https://doi.org/10.1136/vr.100318.

Hettema, J., J. Steele, and W. R. Miller. 2005. Motivational interviewing. Annu. Rev. Clin. Psychol. 1:91-111. https://doi.org/10.1146/ annurev.clinpsy.1.102803.143833.

Imel, Z. E., S. A. Baldwin, J. S. Baer, B. Hartzler, C. Dunn, D. B. Rosengren, and D. C. Atkins. 2014. Evaluating therapist adherence in motivational interviewing by comparing performance with standardized and real patients. J. Consult. Clin. Psychol. 82:472481. https://doi.org/10.1037/a0036158.

Jansen, J. 2010. Mastitis and farmer mindset. Towards effective communication strategies to improve udder health management on Dutch dairy farms. PhD thesis. Wageningen University, Wageningen, the Netherlands.

Jansen, J., and T. J. G. M. Lam. 2012. The role of communication in improving udder health. Vet. Clin. North Am. Food Anim. Pract. 28:363-379. https://doi.org/10.1016/j.cvfa.2012.03.003.

Jansen, J., C. D. Steuten, R. J. Renes, N. Aarts, and T. J. Lam. 2010 Debunking the myth of the hard-to-reach farmer: Effective communication on udder health. J. Dairy Sci. 93:1296-1306. https:// doi.org/10.3168/jds.2009-2794.

Kristensen, A., and C. Enevoldsen. 2008. A mixed methods inquiry: How dairy farmers perceive the value(s) of their involvement in an intensive dairy herd health management program. Acta Vet. Scand. 50:50. https://doi.org/10.1186/1751-0147-50-50.

LeBlanc, S. J., K. D. Lissemore, D. F. Kelton, T. F. Duffield, and K. E. Leslie. 2006. Major advances in disease prevention in dairy cattle. J. Dairy Sci. 89:1267-1279.

Lundahl, B. W.. C. Kunz, C. Brownell, D. Tollefson, and D. L. Burke. 2010. A meta-analysis of motivational interviewing: Twenty-five years of empirical studies. Res. Soc. Work Pract. 20:137-160. https://doi.org/10.1177/1049731509347850.

McArthur, M. L., and J. R. Fitzgerald. 2013. Companion animal veterinarians' use of clinical communications skills. Aust. Vet. J. 91:374-380. https://doi.org/10.1111/avj.12083. 
Rollnick, S., and W. Miller. 1995. What is motivational interviewing? Behav. Cogn. Psychother. 23:325-334. https://doi.org/10.1017/ S135246580001643X.

Miller, W. R., and T. B. Moyers. 2017. Motivational interviewing and the clinical science of Carl Rogers. J. Consult. Clin. Psychol. 85:757-766. https://doi.org/10.1037/ccp0000179.

Miller, W. R., and S. Rollnick. 2009. Ten things that motivational interviewing is not. Behav. Cogn. Psycother. 37:129-140. https:// doi.org/10.1017/S1352465809005128.

Miller, W. R., and S. Rollnick. 2012. Motivational Interviewing. Helping People Change. 3rd ed. Guilford Press, New York.

Moyers, T. B., J. K. Manuel, and D. A. Ernst. 2014. Motivational interviewing treatment integrity coding manual 4.2.1. Accessed Aug. 8, 2019. http://casaa.unm.edu/download/MITI4_2.pdf.

Moyers, T. B., T. Martin, J. K. Manuel, S. M. L. Hendrickson, and W. R. Miller. 2005. Assessing competence in the use of motivational interviewing. J. Subst. Abuse Treat. 28:19-26. https://doi.org/10 $.1016 /$ j.jsat.2004.11.001.

Moyers, T. B., T. Martin, J. K. Manuel, W. R. Miller, and D. A. Ernst. 2010. Revised Global Scales: Motivational Interviewing Treatment Integrity 3.1.1 (MITI 3.1.1). University of New Mexico Center on Alcoholism, Substance Abuse and Addictions (CASAA), Albuquerque, NM.

Moyers, T. B., L. N. Rowell, J. K. Manuel, D. Ernst, and J. M. Houck. 2016. The Motivational Interviewing Treatment Integrity Code (MITI 4): Rationale, preliminary, reliability and validity. J. Subst. Abuse Treat. 65:36-42. https://doi.org/10.1016/j.jsat.2016.01.001.

Radostits, O. M. 2001. Herd Health: Food Animal Production Medicine. 3rd ed. W. B. Saunders Co., Philadelphia, PA.
Ritter, C., C. L. Adams, D. F. Kelton, and H. W. Barkema. 2018. Clinical communication patterns of veterinary practitioners during dairy herd health and production management farm visits. J. Dairy Sci. 101:10337-10350. https://doi.org/10.3168/jds.2018 $-14741$.

Seale, C., C. C. Butler, I. Hutchby, P. Kinnersley, and S. Rollnick. 2007. Negotiating frame ambiguity: A study of simulated encounters in medical education. Commun. Med. 4:177-187. https://do .org/10.1515/CAM.2007.021.

Shaw, J. R., C. L. Adams, B. N. Bonnett, S. Larson, and D. L. Roter. 2008. Veterinarian-client-patient communication during wellness appointments versus appointments related to a health problem companions animal practice. J. Am. Vet. Med. Assoc. 233:15761586 .

Shaw, J. R., B. N. Bonnett, C. L. Adams, and D. L. Roter. 2006. Veterinarian-client-patient communication patterns used during clinical appointment in companion animal practice. J. Am. Vet. Med. Assoc. 228:714-721.

Svensson, C., K. Alvåsen, A. C. Eldh, J. Frössling, and H. Lomander. 2018. Veterinary herd health management - experience among farmers and farm managers in Swedish dairy production. Prev. Vet. Med. 155:45-52. https://doi.org/10.1016/j.prevetmed.2018.04 .012 .

Wickström, H., M. Herzing, L. Forsberg, A. Jacobsson, and H. Källmén. 2017. Applying motivational interviewing to induce compliance with radon gas radiation legislation - A feasibility study. Psychol. Educ. 54:1-22. 